\title{
Hyaluronic acid-coated polymeric micelles with hydrogen peroxide scavenging to encapsulate statins for alleviating atherosclerosis
}

\author{
Dan $\mathrm{Mu}^{1 *}{ }^{*}$, Jianhui $\mathrm{Li}^{2}$, Yu Qi ${ }^{3}$, Xuan Sun ${ }^{3}$, Yihai Liu ${ }^{2}$, Song Shen ${ }^{2}$, Yuyu Li ${ }^{4}$, Biao Xu ${ }^{3,5^{*}}$ and Bing Zhang ${ }^{1,6^{*}}$
}

\begin{abstract}
Inflammation and oxidative stress are two major factors that are involved in the pathogenesis of atherosclerosis. A smart drug delivery system that responds to the oxidative microenvironment of atherosclerotic plaques was constructed in the present study. Simvastatin (SIM)-loaded biodegradable polymeric micelles were constructed from hyaluronic acid (HA)-coated poly(ethylene glycol)-poly(tyrosine-ethyl oxalyl) (PEG-Ptyr-EO) for the purpose of simultaneously inhibiting macrophages and decreasing the level of reactive oxygen species (ROS) to treat atherosclerosis. HA coating endows the micelle system the ability of targeting CD44-positive inflammatory macrophages. Owing to the ROS-responsive nature of PEG-Ptyr-EO, the micelles can not only be degraded by enzymes, but also consumes ROS by itself at the pathologic sites, upon which the accumulation of pro-inflammatory macrophages is effectively suppressed and oxidative stress is alleviated. Consequently, the cellular uptake experiment demonstrated that SIM-loaded HA-coated micelles can be effectively internalized by LPS-induced RAW264.7 cells and showed high cytotoxicity against the cells, but low cytotoxicity against LO2 cells. In mouse models of atherosclerosis, intravenously SIM-loaded HA-coated micelles can effectively reduce plaque content of cholesterol, resulting in remarkable therapeutic effects. In conclusion, the SIM-loaded micelle system provides a promising and innovative option against atherosclerosis.
\end{abstract}

Keywords: Simvastatin, ROS-responsive, Hyaluronic acid, Macrophages, Antioxidative stress

\section{Introduction}

Atherosclerosis is a chronic, systemic inflammatory disease of the large and medium-sized arteries, characterized by the development of plaques due to the retention of lipids in the artery wall and infiltration of leukocytes, which can lead to life-threating events such as myocardial infarction and stroke [1-3]. The inflammatory area

\footnotetext{
*Correspondence: mudan118@126.com; xubiao62@nju.edu.cn; zhangbing_ nanjing@nju.edu.cn

${ }^{1}$ Department of Radiology, Affiliated Nanjing Drum Tower Hospital

of Nanjing University Medical School, 321 Zhongshan road, Nanjing 210008, Jiangsu, China

${ }^{3}$ Department of Cardiology, Affiliated Nanjing Drum Tower Hospital of Nanjing University Medical School, Nanjing 210008, China

Full list of author information is available at the end of the article
}

recruits monocytes, differentiating into macrophages. Upon ingestion of low-density lipoprotein (LDL), macrophages would die or even lead to cellular rupture, recruiting additional immune cells to inflammatory area [4]. Due to the decisive role and abundance in the progression of plaque, macrophages could achieve targeting capacity of plaques. CD44 is one of the specific biomarkers of macrophages and widely expressed on multiple cell types. In inflammatory area, CD44 is upregulated and functionally activated on vascular endothelial, smooth muscle and inflammatory cells. Furthermore, CD44 modulates leukocyte adhesion, migration, and functional phenotype [5]. All these facts make CD44 perfect for a potential therapeutic target of local inflammation at the site of lesion.

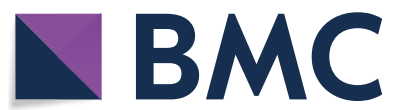

c) The Author(s) 2020. This article is licensed under a Creative Commons Attribution 4.0 International License, which permits use, sharing, adaptation, distribution and reproduction in any medium or format, as long as you give appropriate credit to the original author(s) and the source, provide a link to the Creative Commons licence, and indicate if changes were made. The images or other third party material in this article are included in the article's Creative Commons licence, unless indicated otherwise in a credit line to the material. If material is not included in the article's Creative Commons licence and your intended use is not permitted by statutory regulation or exceeds the permitted use, you will need to obtain permission directly from the copyright holder. To view a copy of this licence, visit http://creativecommons.org/licenses/by/4.0/. The Creative Commons Public Domain Dedication waiver (http://creativecommons.org/publicdomain/zero/1.0/) applies to the data made available in this article, unless otherwise stated in a credit line to the data. 
Statins are widely orally prescribed serum LDL cholesterol-lowering drugs, raising the expression of LDL receptor in hepatocytes [6]. Clinical data has shown that orally administered statins can reduce the risk of cardiovascular disease (CVD) and attenuate the development of atherosclerotic plaques [7]. Statins exhibit pleiotropic properties via reductase derived metabolites blockade, including antioxidant effects, anti-inflammatory effects and multiple vasculoprotective effects on endothelial cells, vascular smooth muscle cells and monocytes [810]. However, considering the low systemic bioavailability, increasing the oral statin dose to attain higher plasma concentrations is not feasible due to the dose-dependent onset of adverse effects such as hepatoxicity and myopathy $[3,6]$. In addition, it was reported that high-dose statins probably induced body weight loss and abnormal serum biomarkers [11]. All these imperfections hinder the further application of statins in the clinical practice.

Increasing researches have demonstrated that the specific delivery of statins to inflammatory atherosclerotic plaques might optimize the therapeutic effects [12-16]. Nanocarriers can target plaques by direct infiltration via the injured endothelium or the dysfunctional neovessels in the adventitia [17]. In addition, nanocarriers administered via intravenous or intraperitoneal injection can be endocytosed by circulating phagocytes, followed by translocation to atherosclerotic lesions by cellular recruitment and infiltration. Whereas nanocarriers only serve as vehicles for targeted delivery of different therapeutics to atherosclerotic plaques in most studies, recent progress also indicated that nanocarriers with intrinsic antioxidant and anti-inflammatory activities are promising next-generation therapies for the treatment of atherosclerosis and other inflammatory diseases [18]. Andrographolide-loaded micelles that responds to the oxidative microenvironment of atherosclerotic plaques was constructed based on the block copolymer of poly(ethylene glycol) and poly(propylene sulphide) (PEG-PPS) for the purpose of simultaneously decreasing inflammatory response and the level of reactive oxygen species (ROS) to treat atherosclerosis [19]. Andrographolide exhibits great anti-inflammatory activity and the antioxidant activity of PEG-PPS was endowed by the thioether in the hydrophobic block PPS. Another research has reported a novel dual ROSsensitive and CD44 receptors targeting nanocarriers composed of oligomeric hyaluronic acid- $2^{\prime}$-[propane-2, 2-diyllbls (thio)] diacetic acl-hydroxymethylferrocene (HASF) [20]. Curcumin was encapsulated into HASF and provided potent anti-inflammatory and antioxidant effects. In addition, 2'-[propane-2, 2-diyllbls (thio)] diacetic acl (TKL) and hydroxymethylferrocene (Fc) acted as ROS-responsive linkers and also enhanced antioxidant effects. Another ROS-responsive delivery system combining anti-inflammatory and antioxidant effects has been constructed based on arylboronic esters-modified amphiphilic oxidation-sensitive chitosan oligosaccharide (Oxi-COS) [4]. Except for the above, peroxalate ester is another ROS-responsive bonds and has been used to establish drug delivery systems [21-23]. However, as far as the information goes, it has not been reported that peroxalate esters are used to achieve the combination of anti-oxidation and antiinflammation in the treatment of atherosclerosis.

Herein, SIM-loaded hyaluronic acid (HA)-coated biodegradable polymeric micelles assembled from an amphiphilic diblock copolymer poly(ethylene glycol)poly(tyrosine-ethyl oxalyl) (PEG-Ptyr-EO) were established for atherosclerosis treatment. The preparation route of SIM-loaded polymeric micelles was depicted in Scheme 1. SIM-loaded polymeric micelles were prepared by self-assembly and HA coating on the surface of micelles was achieved by electrostatic interaction. The hydrophobic block Ptyr-EO can react with $\mathrm{H}_{2} \mathrm{O}_{2}$ and this process takes place only at the pathologic sites where the $\mathrm{H}_{2} \mathrm{O}_{2}$ concentration is far above that in normal tissues, and thereby the drug delivery efficiency and therapeutic efficacy may be dramatically increased. Furthermore, SIM and the polymers serve to reduce inflammation and $\mathrm{H}_{2} \mathrm{O}_{2}$ levels, respectively, both of which are essential in the formation and progression of atherosclerosis. It is anticipated that, SIM and the drug carrier itself may exert a synergistic effect for effective treatment of atherosclerosis.

\section{Methods \\ Materials}

Maleimide poly(ethylene glycol) amine (Mal-PEG$\mathrm{NH}_{2}, 5000 \mathrm{Da}$ ) was obtained from Xi'an Ruixi Biological Technology Co., Ltd. Hyaluronic acid (HA, 40-100 kDa), cysteamine hydrochloride and ethyl oxalyl chloride were purchased from Shanghai McLean Biochemical Technology Co., Ltd. L-Tyrosine $N$-carboxyanhydride (Tyr-NCA) was brought from Shanghai Bide Pharmaceutical Technology Co., Ltd. Lipopolysaccharide (LPS) was brought from Sigma-Aldrich. Cy5 NHS ester was purchased from Shanghai Yuanye Biotechnology Co., Ltd. 2', 7'-Dichlorofluorescin diacetate (DCFH-DA) was obtained from Beyotime Institute of Biotechnology. Anhydrous $\mathrm{N}, \mathrm{N}$-dimethylformamide (DMF) and dichloromethane (DCM) were purchased from Energy Chemical. Other solvents, including dimethylsulfoxide (DMSO), tetrahydrofuran (THF) and triethylamine (TEA) were brought from Greagent and used as received. 


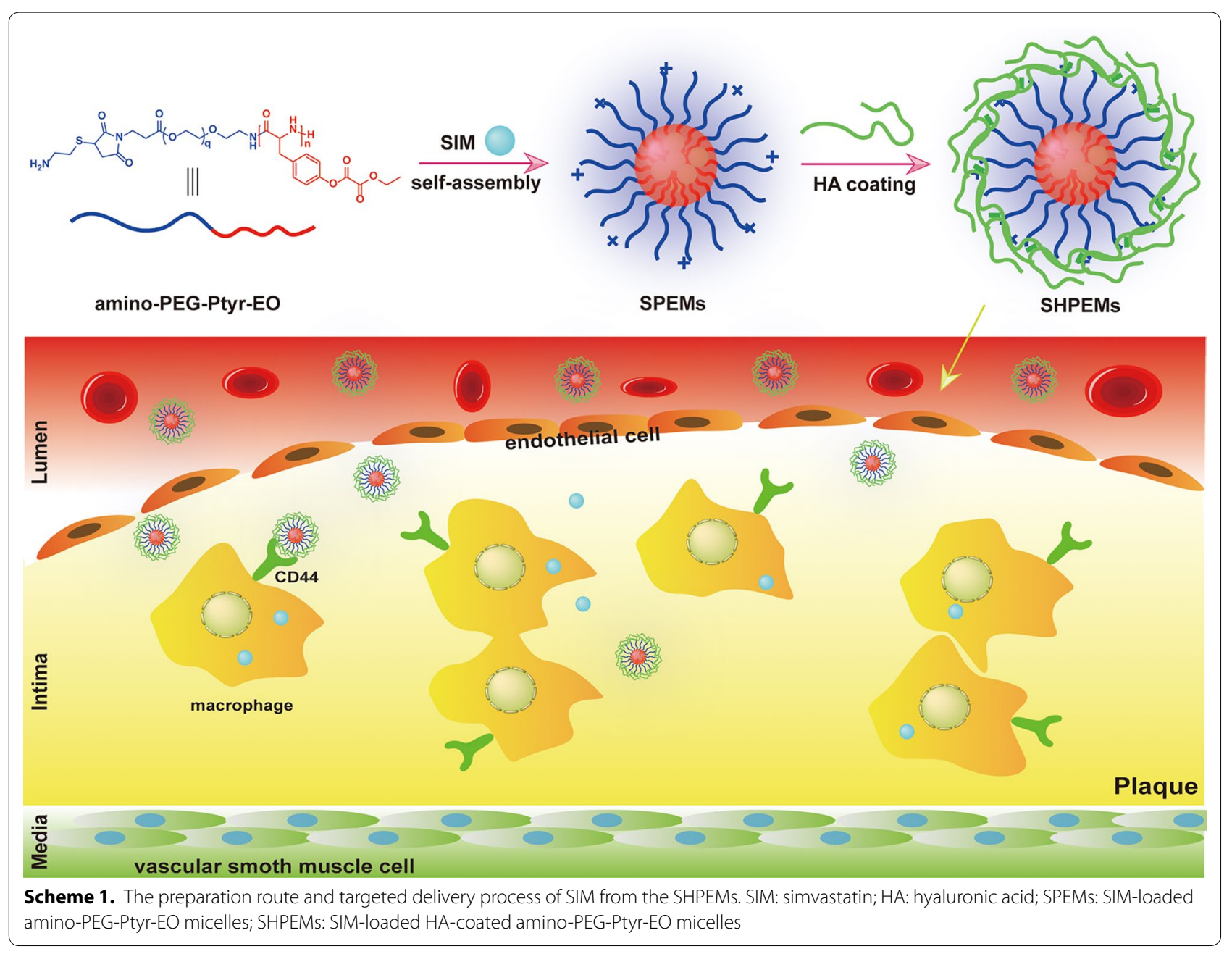

\section{Synthesis of Mal-PEG-Ptyr}

Mal-PEG-Ptyr was synthesized according to the report [24, 25]. Briefly, Mal-PEG- $\mathrm{NH}_{2}(1.0 \mathrm{~g}, 0.2 \mathrm{mmol})$ and Tyr-NCA (1.0 g, $4.8 \mathrm{mmol})$ were dissolved in anhydrous $15 \mathrm{~mL}$ DMF. The reaction was performed at $35^{\circ} \mathrm{C}$ under $\mathrm{N}_{2}$ for $72 \mathrm{~h}$. The reaction mixture was concentrated under vacuum and poured into excess cold diethyl ether for five times. The precipitation was collected and dried under vacuum.

${ }^{1} \mathrm{H}$ NMR (500 MHz, DMSO- $\left.d_{6}\right)$ ): $9.09(\mathrm{~s}, 23.81 \mathrm{H},-$ $\mathrm{OH}), 7.92(\mathrm{~m}, 21.93 \mathrm{H},-\mathrm{CONH}-)$, 7.08-6.56 (m, 105.02 $\left.\mathrm{H},-\mathrm{C}_{6} \boldsymbol{H}_{4}-\right), 4.43(\mathrm{~m}, 20.44 \mathrm{H},-\mathrm{CH}-), 3.56(\mathrm{~m}, 450.00$ $\left.\mathrm{H},-\mathrm{CH}_{2} \mathrm{CH}_{2}-\right)$, 2.89-2.59(m, $\left.44.80 \mathrm{H},-\mathrm{CH}_{2} \mathrm{C}_{6} \mathrm{H}_{4}-\right)$.

\section{Synthesis of Mal-PEG-Ptyr-EO}

Mal-PEG-Ptyr (0.5 g) was dissolved in anhydrous DMF. TEA $(0.6 \mathrm{~mL})$ was added into DMF solution. Ethyl oxalyl chloride $(0.3 \mathrm{~g}, 2.2 \mathrm{mmol})$ in DCM was added dropwise in ice bath. The reaction was stirred for $24 \mathrm{~h}$. After that, the reaction mixture was filtrated and washed by saturated $\mathrm{NaCl}$ solution for three times. The organic phase was collected and poured into excess cold diethyl ether for three times. The precipitation was collected and dried under vacuum.

${ }^{1} \mathrm{H}$ NMR $\left(500 \mathrm{MHz}, \mathrm{DMSO}-d_{6}\right) \delta: 7.49-6.73(\mathrm{~m}, 85.98$ $\left.\mathrm{H},-\mathrm{C}_{6} \boldsymbol{H}_{4}-\right), 4.31\left(\mathrm{~m}, 40.11 \mathrm{H},-\mathrm{CH}_{2} \mathrm{CH}_{3}\right), 3.56(\mathrm{~m}$, $\left.450.00 \mathrm{H},-\mathrm{CH}_{2} \mathrm{CH}_{2}-\right), 1.30\left(\mathrm{~m}, 63.53 \mathrm{H},-\mathrm{CH}_{2} \mathrm{CH}_{3}\right)$.

\section{Synthesis of amino-PEG-Ptyr-EO}

Mal-PEG-Ptyr-EO $(0.3 \mathrm{~g})$ was dissolved in $10 \mathrm{~mL}$ DMF with cysteamine hydrochloride $(0.1 \mathrm{~g})$ added. The reaction was stirred at room temperature for $24 \mathrm{~h}$. After that, the mixture was diluted with DCM, washed with saturated $\mathrm{NaCl}$ solution for three times and dried with anhydrous $\mathrm{Na}_{2} \mathrm{SO}_{4}$. After concentration, the mixture was poured into cold diethyl ether. Amino-PEG-Ptyr-EO was obtained by filtration and drying under vacuum. To label amino-PEG-Ptyr-EO with fluorescence dye, $10 \mathrm{mg}$ of Cy5 NHS ester was reacted with $50 \mathrm{mg}$ of amino-PEG-PtyrEO to generate Cy5-PEG-Ptyr-EO. 
${ }^{1} \mathrm{H}$ NMR $\left(500 \mathrm{MHz}\right.$, DMSO- $\left.d_{6}\right) \delta: 7.44-6.81(\mathrm{~m}, 91.77$ $\left.\mathrm{H},-\mathrm{C}_{6} \boldsymbol{H}_{4}-\right), 4.32\left(\mathrm{~m}, 40.21 \mathrm{H},-\mathrm{CH}_{2} \mathrm{CH}_{3}\right), 3.56(\mathrm{~m}$, $\left.450.00 \mathrm{H},-\mathrm{CH}_{2} \mathrm{CH}_{2}-\right), 1.29\left(\mathrm{~m}, 61.77 \mathrm{H},-\mathrm{CH}_{2} \mathrm{CH}_{3}\right)$.

\section{$\mathrm{H}_{2} \mathrm{O}_{2}$ degradation}

Mal-PEG-Ptyr-EO was dissolved completely in DMSO- $d_{6}$ with addition of $50 \mathrm{mM} \mathrm{H}_{2} \mathrm{O}_{2}$ in a nuclear magnetic tube. The tube was incubated at $37^{\circ} \mathrm{C}$ for $30 \mathrm{~min}, 60 \mathrm{~min}$ and $120 \mathrm{~min}$. The chemical structure was characterized by ${ }^{1} \mathrm{H}$ NMR. The amount of $\mathrm{H}_{2} \mathrm{O}_{2}$ was detected after incubation for $30 \mathrm{~min}, 60 \mathrm{~min}$ and $120 \mathrm{~min}$ by hydrogen peroxide assay kit (Beyotime).

\section{Preparation of micelles}

Before preparing micelles, the molecular weights and their corresponding distribution of polymers were measured by the gel permeation chromatography system (GPC, Waters). THF was used as the eluent at a low flow rate of $1.0 \mathrm{~mL} / \mathrm{min}$. The standard curve was constructed by monodispersed polystyrene standard. The critical micelle concentrations (CMC) for the block copolymers were determined using pyrene as the probe [26]. Briefly, a constant concentration of pyrene $\left(5.0 \times 10^{-7} \mathrm{~mol} / \mathrm{L}\right)$ was incubated with varying concentrations of lyophilized block copolymer $(0.05-51.2 \mu \mathrm{g} / \mathrm{mL})$ in phosphate buffer solution (PBS) at $37{ }^{\circ} \mathrm{C}$ for $36 \mathrm{~h}$. All samples were run with a total sample volume of $1.2 \mathrm{~mL}$ in a quartz cuvette. The spectrophotometer (Hengping F9600) was used to record the excitation spectra for each polymer concentration at 338 and $333 \mathrm{~nm}$. A plot of the $I_{338} / I_{333}$ ratio versus log of the block copolymer concentration was drawn to calculate the CMC.

Micelles based on amino-PEG-Ptyr-EO were prepared by dialysis method. Briefly, $10 \mathrm{mg}$ amino-PEG-Ptyr-EO dissolved in $1 \mathrm{~mL}$ DMSO completely was placed into a dialysis tube $\left(M_{\mathrm{w}} 3500\right)$ and dialyzed against pure water for $24 \mathrm{~h}$. Amino-PEG-Ptyr-EO micelles (PEMs) were obtained by lyophilization. HA-coated PEMs (HPEMs) were prepared by electrostatic attraction. Briefly, PEMs suspension was slowly added to a HA solution under vigorous stirring at room temperature. The mass ration of HA to PEMs and the concentration of HA were further investigated to obtain the optimum formulation. The method to prepare Cy5-labeled micelles was as mentioned above, except that amino-PEG-Ptyr-EO was replaced with amino-PEG-Ptyr-EO/Cy5-PEG-Ptyr-EO $(95 / 5, \mathrm{w} / \mathrm{w})$.

\section{Characterization of micelles}

The size distribution and Zeta potential of micelles were recorded by dynamic light scattering (DLS, Malvern Zetasizer). The morphologies of micelles were investigated by transmission electron microscopy (TEM,
JEM-2100). In brief, a drop of micelle suspension was placed onto the surface of the copper grid. After stained for $5 \mathrm{~min}$, the suspension on the copper grid was absorbed by filter paper. The TEM photos were taken after air-drying.

\section{Drug encapsulation and release}

SIM-loaded PEMs (SPEMs) were prepared by dialysis method. Briefly, $100 \mathrm{mg}$ amino-PEG-Ptyr-EO and $10 \mathrm{mg}$ SIM were dissolved in $2 \mathrm{~mL}$ DMSO completely. Then, the mixture was placed into a dialysis tube $\left(M_{\mathrm{w}} 3500\right)$ and dialyzed against pure water for $48 \mathrm{~h}$ to remove DMSO and unloaded SIM. After that, the suspension was filtrated through $0.45 \mu \mathrm{m}$ syringe filter. Afterwards, SIMloaded HPEMs (SHPEMs) were obtained by HA coating with $0.5 \mathrm{mg} / \mathrm{mL}$ HA solution with the mass ratio of $3 / 1$ (HA to SPEMs). Besides, SIM-loaded Mal-PEG-Ptyr micelles (SPMs) were prepared by the same method and used in drug release experiments. SHPEMs were incubated in PBS (pH 7.4) and DMEM with 10\% FBS for 16 days. The average size was measured at predetermined time points.

To determine the drug loading efficiency (DLE) and drug loading content (DLC), a known amount of SPEMs was dissolved in $1 \mathrm{~mL}$ of THF. This solution was then analyzed by an ultraviolet (UV) spectrophotometer at $238 \mathrm{~nm}$ with PEMs suspension as the control [27]. The drug concentration in the solution was calculated based on the standard curve. DLE and DLC were obtained in accordance with the following formulae:

$$
\begin{aligned}
& D L C \%=\frac{\text { weight of SIM loaded }}{\text { weight of SIM loaded micelles }} \times 100 \% \\
& D L E \%=\frac{\text { weight of SIM loaded }}{\text { weight of SIM in feeding }} \times 100 \%
\end{aligned}
$$

The drug release was conducted based on SPEMs and SPMs. Briefly, $1 \mathrm{~mL}$ of SPEMs or SPMs suspension with the same SIM amount were placed into the dialysis bag $\left(M_{\mathrm{w}} 3500\right)$ suspended in $20 \mathrm{~mL}$ PBS (pH 7.4) containing $0.2 \%$ SDS with or without $10 \mathrm{mM} \mathrm{H}_{2} \mathrm{O}_{2}$. The sealed vials were shaken at $37{ }^{\circ} \mathrm{C}$ for $72 \mathrm{~h}$. At predetermined time intervals, the release medium was withdrawn to measure the amount of SIM by UV method and $20 \mathrm{~mL}$ fresh medium was added. The experiment was performed in triplicates. After the experiment, the residues of SHPEMs were collected to measure the size distribution and morphology by DLS and TEM.

\section{Cell culture}

RAW264.7 and LO2 cells were employed to evaluate the cellular uptake and cytotoxicity against SIM and 
SIM-loaded micelles. RAW264.7 cells, highly expressed CD44 receptors, were cultured in Dulbecco's modified eagle's medium (DMEM) containing 10\% FBS, $100 \mathrm{mg} /$ $\mathrm{mL}$ of penicillin and $100 \mathrm{mg} / \mathrm{mL}$ of streptomycin at $37{ }^{\circ} \mathrm{C}$ in an incubator with $5 \% \mathrm{CO}_{2}$ humid atmosphere. To induce inflammatory reaction, RAW264.7 cells were simulated with $100 \mathrm{ng} / \mathrm{mL}$ LPS for $24 \mathrm{~h}$. LO2, negatively expressed CD44 receptors, cells were cultured in RPMI 1640 supplemented with $10 \% \mathrm{FBS}, 100 \mathrm{mg} / \mathrm{mL}$ of penicillin and $100 \mathrm{mg} / \mathrm{mL}$ of streptomycin at $37^{\circ} \mathrm{C}$ in an incubator with $5 \% \mathrm{CO}_{2}$ humid atmosphere.

\section{Cellular uptake}

LPS-simulated RAW264.7 cells were reported to highly express CD44 receptors that can specifically bind HA ligands. However, $\mathrm{LO} 2$ cells were reported to negatively express CD44 receptors [28]. Therefore, RAW264.7 and LO2 cells were seeded into glass plates with the density of $1.0 \times 10^{5}$ cells/plate. After incubation for $12 \mathrm{~h}$, the culture medium was replaced with fresh medium containing Cy5-labeled PEMs or HPEMs with or without HA pretreatment. After incubation for another $2 \mathrm{~h}$, cells were washed with PBS for two times and fixed with $4 \%$ paraformaldehyde for $15 \mathrm{~min}$. After stained with DAPI for $10 \mathrm{~min}$, fluorescence images of cells were obtained using a confocal laser scanning microscope (CLSM, Leica).

\section{Cytotoxicity}

The cytotoxicity of SIM and SIM-loaded micelles against RAW264.7 or LO2 cells was measured by MTT assay. Briefly, LPS-simulated RAW264.7 cells were seeded at a density of $4 \times 10^{3}$ cells/well in 96 -well plates. When the cells reached $80 \%$ confluence, blank micelles, free SIM, SPEMs or SHPEMs at different SIM concentrations $(0.25$, $0.5,1.0,2.0,4.0$, and $8.0 \mu \mathrm{g} / \mathrm{mL}$ ) or different polymer concentrations $(50,100,200,300,400$ and $500 \mu \mathrm{g} / \mathrm{mL})$ in DMEM were added and cultured for $24 \mathrm{~h}$. LO2 cells were seeded and cultured as the same method, and treated with free SIM and SHPEMs (SIM: 0.25, 0.5, 1.0, 2.0, 4.0, and $8.0 \mu \mathrm{g} / \mathrm{mL}$ ) for $24 \mathrm{~h}$. To evaluate the time-dependent cytotoxicity, cells were incubated with free SIM and SHPEMs (SIM: $4.0 \mu \mathrm{g} / \mathrm{mL}$ ) for $24 \mathrm{~h}, 48 \mathrm{~h}$ and $72 \mathrm{~h}$. To survey the influence of HA coating on the cytotoxicity, cells were incubated with free SIM, SPEMs and SHPEMs at the same SIM concentration $(8.0 \mu \mathrm{g} / \mathrm{mL})$ for $4 \mathrm{~h}$. Then, the culture medium was replaced with fresh medium and cells were incubated for another $20 \mathrm{~h} .10 \mu \mathrm{L}$ of $5 \mathrm{mg} / \mathrm{mL}$ MTT solution was added to each well and the cells were incubated for another $4 \mathrm{~h}$. Subsequently, the medium was carefully removed and $100 \mu \mathrm{L}$ of DMSO was added to each well. The absorbance of each well was recorded on a microplate reader at the wavelength of $562 \mathrm{~nm}$. The experiment was performed in triplicates.

$$
\text { Cell viability } \%=\frac{O D_{\text {sample }}-O D_{\text {blank }}}{O D_{\text {control }}-O D_{\text {blank }}} \times 100 \%
$$

where $\mathrm{OD}_{\text {sample }}$ means the $\mathrm{OD}$ value from wells treated with drugs, $\mathrm{OD}_{\text {control }}$ from wells treated with DMEM medium and $\mathrm{OD}_{\text {blank }}$ from wells without cells but culture medium.

\section{ROS generation}

DCFH-DA was used to evaluate the generation of intracellular ROS by fluorescence imaging and flow cytometry. In brief, RAW264.7 and LPS-induced RAW 264.7 cells were seeded in a 6-well plate and cultured for $12 \mathrm{~h}$ at $37{ }^{\circ} \mathrm{C}$. Next, cells were incubated with DCFH-DA $(10 \mu \mathrm{M})$ for $30 \mathrm{~min}$. Then the cells were washed with PBS and incubated with different samples respectively (PBS, free SIM, HPEMs and SHPEMs). After incubation for another $12 \mathrm{~h}$, the cells were washed with PBS to remove free samples, following by observation using a fluorescence microscopy and analysis by flow cytometry.

\section{Aminals treatment}

Apolipoprotein E-deficient $\left(\mathrm{ApoE}^{-1-}\right)$ mice (six-week old) were obtained from the Nanjing University in Nanjing, China. All the animal care and experimental protocols were carried out with review and approval from the Laboratory Animal Welfare and Ethics Committee of the Nanjing University. ApoE ${ }^{-1-}$ mice were fed with a highfat diet for 8 weeks and treated once a week with saline, free SIM, SPEMs and SHPEMs at a dose of $30 \mathrm{mg} / \mathrm{kg}$ for SIM.

\section{ORO and H\&E staining}

After treatment for 4 weeks, the mice were sacrificed, and the aortas and major organs were harvested. Aortas, from the heart to the iliac bifurcation, were fixed by perfusion with paraformaldyhyde ( $4 \%$ in PBS). After removing the periadventitial tissue, aortas were dissected longitudinally and stained with Oil Red O (ORO) to quantify the lesion areas of the plaques by NIH ImageJ software. Besides, sections of the major organs including heart, liver, spleen, lung, and kidney were also analyzed by hematoxylin-eosin (H\&E) staining.

\section{Results and discussion}

\section{Synthesis of polymers}

As shown in Scheme 2, Mal-PEG-Ptyr copolymers were obtained via the ring-opening polymerization of tyrosine $\mathrm{N}$-carboxyanhydride (Tyr-NCA) using Mal-PEG- $\mathrm{NH}_{2}$ as the initiator (Scheme 1). The ${ }^{1} \mathrm{H}$ NMR spectrum of MalPEG-Ptyr displays the characteristic signals of PEG (peak $\boldsymbol{a}, \delta$ 3.56) and phenolic hydroxyl groups (peak $\boldsymbol{f}, \delta$ 9.09) (Additional file 1: Figure S1A). Other signals of Ptyr were 


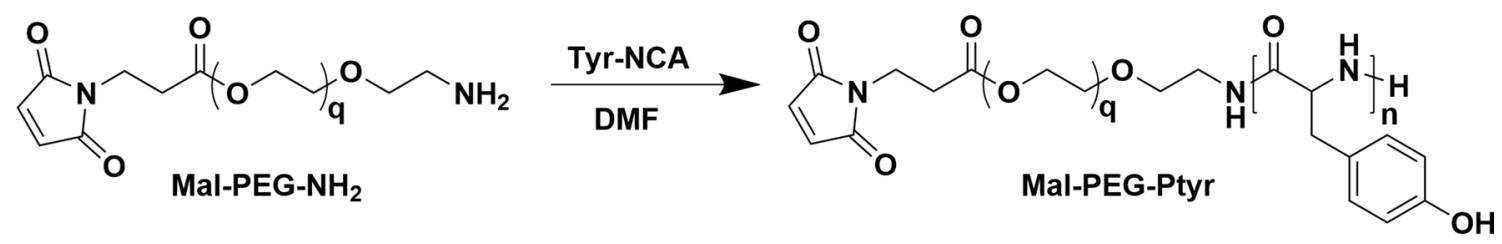<smiles>CCOC(=O)C(=O)NC(Cc1ccc(OC(=O)C(=O)OCC)cc1[18OH])C(=O)NCCOCCOC(=O)CCN1C(=O)C=CC1=O</smiles><smiles>CCOC(=O)C(=O)Oc1ccc(CC(NCCOC(C)(Cl)C(=O)NCCOC(=O)CCN2C(=O)CC(SCCN)C2=O)C(=O)NCCCS)cc1</smiles>

Scheme 2 Schematic depiction of the synthetic route of amino-PEG-Ptyr-EO

Table 1 Molecular weight of copolymers

\begin{tabular}{llll}
\hline Copolymer & \multicolumn{1}{c}{$\boldsymbol{M}_{\mathbf{n}}(\mathbf{k g} / \mathbf{m o l})$} & & $\boldsymbol{M}_{\mathbf{w}} \mathbf{M}_{\mathbf{n}}^{\mathbf{b}}$ \\
\cline { 2 - 3 } & $\mathbf{1}^{\mathbf{H}} \mathbf{N M R}$ & \\
\hline Mal-PEG-Ptyr & $8.3-9.2$ & $\mathbf{G P C}^{\mathbf{b}}$ & \\
Mal-PEG-Ptyr-EO & $10.7-11.3$ & 10.6 & 1.21 \\
\hline
\end{tabular}

a Calculated from ${ }^{1} \mathrm{H}$ NMR (DMSO- $d_{6}$ ); ${ }^{\text {b }}$ determined by GPC

labeled and attributed to benzene (peak $\boldsymbol{d}$ and $\boldsymbol{e}, \delta 6.96$ and 6.60), methylene (peak $c, \delta 2.84-2.66$ ) and methyne (peak $\boldsymbol{b}, \delta 4.43$ ), respectively. The integral area of these signal peaks was calculated and listed in Additional file 1: Figure S1A. The $M_{\mathrm{n}}$ of Mal-PEG-Ptyr block copolymers was calculated by comparing the integrals of peak $\boldsymbol{a}$ and peak $\boldsymbol{c}$, or peak $\boldsymbol{e}$. The $M_{\mathrm{n}}$ of Mal-PEG-Ptyr was also determined by GPC as shown in Table 1 and the trace of GPC was demonstrated in Additional file 1: Figure S2. GPC measurements suggested that Mal-PEG-Ptyr had a narrow molecular weight distribution.

Mal-PEG-Ptyr-EO was synthesized by conjugation of tyrosine units with ethyl oxalyl chloride. The ${ }^{1} \mathrm{H}$ NMR spectrum of Mal-PEG-Ptyr-EO was exhibited in Additional file 1: Figure S1B. The emerging signals assigned to ethyl oxalyl esters were listed and labeled as methylene (peak $\boldsymbol{g}, \delta 4.31$ ) and methyl groups (peak $\boldsymbol{h}, \delta 1.30$ ) that indicated the successful synthesis of Mal-PEG-Ptyr-EO.
The integrals of peak $\boldsymbol{g}$ and $\boldsymbol{h}$ were calculated and compared with these of peak $\boldsymbol{a}$, suggesting that ca. 20 tyrosine units were modified by ethyl oxalyl chloride, which was evidenced by the increase of the molecular weight of Mal-PEG-Ptyr-EO in Table 1. Notably, after modification, the positions of signals assigned to Ptyr had changed (labeled as peak $\boldsymbol{b}^{\prime}, \boldsymbol{c}^{\prime}, \boldsymbol{d}^{\prime}$ and $\boldsymbol{e}^{\prime}$ ). At last, cysteamine was conjugated with Mal-PEG-Ptyr-EO to give amine-PEGPtyr-EO. The ${ }^{1} \mathrm{H}$ NMR spectrum was demonstrated in Additional file 1: Figure S1C. The emerging peak $\boldsymbol{i}(\delta$ 3.17) indicated the successful conjugation.

\section{$\mathrm{H}_{2} \mathrm{O}_{2}$ degradation}

It has been reported that peroxalate ester can be oxidized rapidly by $\mathrm{H}_{2} \mathrm{O}_{2}$ to generate $\mathrm{CO}_{2}$ [23]. Hence, the polymer with peroxalate ester linkages serve as a new family of biocompatible and biodegradable materials, which is applied to deliver therapeutic drugs to inflammation sites or tumor tissues that overproduce ROS species. Theoretically, Mal-PEG-Ptyr-EO can be oxidized into MalPEG-Ptyr, $\mathrm{CO}_{2}$ and alcohol as shown in Additional file 1: Figure S3A. To prove it, Mal-PEG-Ptyr-EO was incubated with $50 \mathrm{mM} \mathrm{H}_{2} \mathrm{O}_{2}$ for different time and the chemical structures of polymers were monitored as shown in Additional file 1: Figure S3B. After incubation for $30 \mathrm{~min}$, the signal peak $\boldsymbol{h}(\delta 1.30)$ turned into peak $\boldsymbol{h}^{\prime}(\delta 1.21)$ and the signal of peak $g$ decreased, indicating the generation 
of alcohol. After incubation for $60 \mathrm{~min}$ and $120 \mathrm{~min}$, peak $\boldsymbol{g}$ disappeared completely. Besides, peak $\boldsymbol{g}^{\prime}$ was probably hidden by the signal of PEG, which makes it cannot be observed. The degradation of Mal-PEG-Ptyr-EO by $\mathrm{H}_{2} \mathrm{O}_{2}$ was also proved by the consumption of $\mathrm{H}_{2} \mathrm{O}_{2}$ as shown in Additional file 1: Figure S4. In the first $60 \mathrm{~min}, \mathrm{H}_{2} \mathrm{O}_{2}$ was gradually consumed. However, in the second $60 \mathrm{~min}$, the amount of $\mathrm{H}_{2} \mathrm{O}_{2}$ was almost the same, indicating complete oxidation of Mal-PEG-Ptyr-EO.

\section{Micelle preparation and characterization}

Before preparing micelles, the CMC of Mal-PEG-Ptyr and Mal-PEG-Ptyr-EO was determined using pyrene as a fluorescence probe. Notably, Mal-PEG-Ptyr-EO displayed a CMC of $3.4 \mu \mathrm{g} / \mathrm{mL}$, lower than that of MalPEG-Ptyr $(8.9 \mu \mathrm{g} / \mathrm{mL})$ as shown in Fig. 1a, which was possibly caused by the modification of ethyl oxalyl chloride to improve the hydrophobicity of Ptyr moieties. Micelles based on amine-PEG-Ptyr-EO were prepared
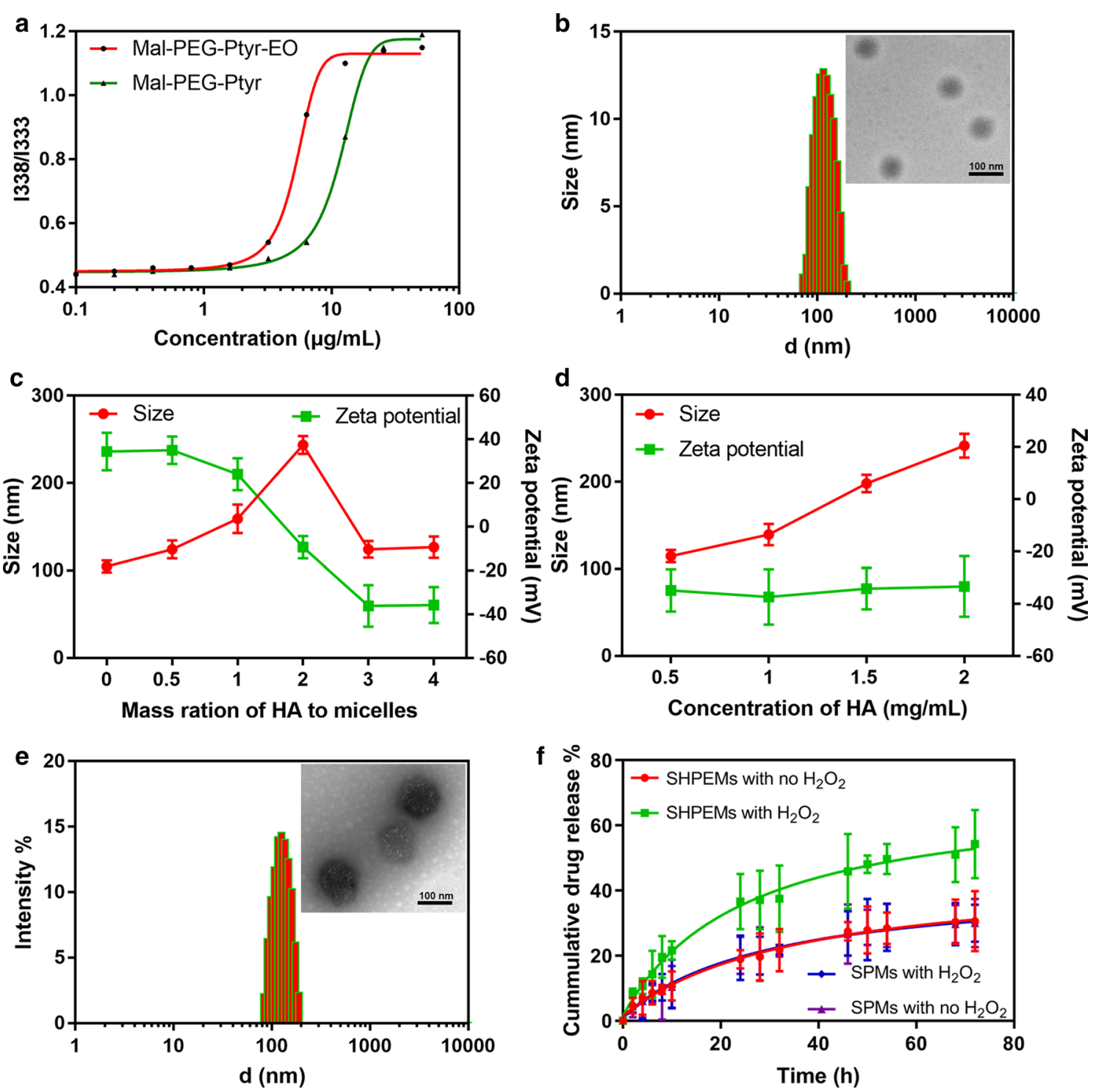

Fig. 1 Determination of CMC of Mal-PEG-Ptyr and Mal-PEG-Ptyr-EO (a); size distribution and morphology of amino-PEG-Ptyr-EO micelles (b); the changes of HA-coated amino-PEG-Ptyr-EO micelles with different mass ratio of HA to amino-PEG-Ptyr-EO micelles (c); the changes of HA-coated amino-PEG-Ptyr-EO micelles with different concentration of HA (d); size distribution and morphology of HA-coated amino-PEG-Ptyr-EO micelles with $0.5 \mathrm{mg} / \mathrm{mL} \mathrm{HA}$ and 3/1 mass ratio of HA to amino-PEG-Ptyr-EO micelles (e); SIM release from SPMs and SPEMs in pH 7.4 PBS with or without $10 \mathrm{mM} \mathrm{H}_{2} \mathrm{O}_{2}$ (f). SIM simvastatin, HA hyaluronic acid, SPEMs SIM-loaded amino-PEG-Ptyr-EO micelles, SHPEMs SIM-loaded HA-coated amino-PEG-Ptyr-EO micelles, SPMs SIM-loaded Mal-PEG-Ptyr micelles, SPEMs SIM-loaded amino-PEG-Ptyr-EO micelles 
by dialysis method. The size distribution and morphology were detected by DLS and TEM in Fig. 1b. It showed an average size of $131.5 \pm 6.4 \mathrm{~nm}$ with PDI $0.106 \pm 0.012$. Besides, the micelles exhibited Zeta potential of $38.4 \pm 9.7 \mathrm{mV}$, resulting from free amino groups. HA with molecular weight of $40-100 \mathrm{kDa}$ was coated on the surface of micelles by electrostatic interaction with amino groups. As shown in Fig. 1c, the size increased and the Zeta potential decreased at the beginning with the addition of HA. However, the size decreased rapidly and the Zeta potential continued to decrease when the mass ratio of HA to amine-PEG-Ptyr-EO micelles increased to 3/1, which indicated the complete coating of HA. To explore the influence of the concentration of HA, micelles were coated with HA with concentration ranging from 0.5 to $2 \mathrm{mg} / \mathrm{mL}$. As shown in Fig. 1d, with the increase of HA concentration, the average size of coated micelles gradually increased, while the Zeta potential was almost unchanged. Figure 1e showed the size distribution and morphology of HA-coated micelles with the mass ratio of HA $(0.5 \mathrm{mg} / \mathrm{mL})$ to micelles of $3 / 1$. It demonstrated uniform spherical micelles with size around $142.3 \mathrm{~nm}$.

\section{Drug loading and release}

During the formation of micelles, SIM and the hydrophobic segments of PEG-Ptyr-EO were forced to aggregate by $\mathrm{H}_{2} \mathrm{O}$ molecules. Thus, loaded drugs enlarged the core of the micelles. The average size of SHPEMs was higher than that of HPEMs (Additional file 1: Figure S5A), indicating successful encapsulation of SIM. After SIM was loaded into micelles, the average size of SHPEMs was measured in PBS (pH 7.4) and DMEM containing 10\% FBS. As shown in Additional file 1: Figure S5B, SHPEMs can remain stable in PBS and DMEM for at least half month, indicating great colloidal stability. The DLC and DLE of SPEMs were calculated as 7.3\% and $84.3 \%$, respectively. The in vitro drug release profiles of SHPEMs and SPMs in $\mathrm{pH}$ 7.4 PBS were evaluated by the dialysis method. As depicted in Fig. 1f, sustained release of SIM from SPEMs or SPMs can be maintained for over $72 \mathrm{~h}$. Fast SIM release occurred within initial $24 \mathrm{~h}$. It was noteworthy that the release profiles of SIM from SPMs and SPEMs without $\mathrm{H}_{2} \mathrm{O}_{2}$ addition were quite similar. Notably, SIM release from SPEMs with $\mathrm{H}_{2} \mathrm{O}_{2}$ addition was obviously faster, which was possibly caused by the oxidation of peroxalate esters. Specifically, the accumulative release amount of SIM from SPEMs with $\mathrm{H}_{2} \mathrm{O}_{2}$ addition was $54.3 \%$ for $72 \mathrm{~h}$, which it was $30.6 \%$ without $\mathrm{H}_{2} \mathrm{O}_{2}$ addition. According to the profile of drug release, it can be concluded that SIM was gradually release from micelles and no obvious "sudden release" phenomenon was observed, indicating SIM was mainly encapsulated in the hydrophobic core of micelles. After incubation with
$\mathrm{H}_{2} \mathrm{O}_{2}$ for $72 \mathrm{~h}$, the size distribution and morphology of SHPEMs were measured as shown in Additional file 1: Figure S6. SHPEMs became anomalous aggregates with widened size distribution, which was possibly caused by the oxidation of peroxalate esters and drug release.

\section{Cellular uptake and cytotoxicity}

To investigate CD44-mediated cellular uptake, LPSinduced RAW264.7 and LO2 (less CD44 expression) were incubated with different micelle formulations. Micelles were labeled with Cy5. As shown in Additional file 1: Figure S7, labeling with Cy5 hardly changed the size distribution and morphology of PEMs. As shown in Fig. 2a, both RAW264.7 and LO2 cells exhibited significant $\mathrm{Cy} 5$ fluorescence after treated with different micelles. Notably, cells treated with Cy5-labeled HPEMs exhibited stronger fluorescence than Cy5-labeled PEMs. However, after cells were pre-treated with free HA and then incubated with Cy5-labeled HPEMs, the red fluorescence decreased significantly. Moreover, LO2 exhibited weak Cy5 fluorescence after treatment with Cy5-labeled HPEMs. These results indicated the CD44-mediated endocytosis of LPS-induced RAW264.7 against HAcoated micelles.

To study the cytotoxicity of SIM-loaded micelles, the cell viability was tested by MTT assay. Firstly, both PEMs and HPEMs exhibited the cell viability over $90 \%$ at all tested concentrations, indicating great biocompatibility (Additional file 1: Figure S8). Both free SIM and SHPEMs showed comparative cell viability of 24 h's incubation against RAW264.7 cells. Free SIM showed slightly higher cytotoxicity than SHPEMs at all tested SIM concentrations (Fig. 2b). Interestingly, the cell viabilities of both free SIM and SHPEMs against LO2 cells were over 90\%, indicating great biosafety (Additional file 1: Figure S9). To survey time-dependent cytotoxicity, LPS-induced RAW264.7 cells were incubated free SIM and SHPEMs with $4 \mu \mathrm{g} / \mathrm{mL}$ SIM for $24 \mathrm{~h}, 48 \mathrm{~h}$ and $72 \mathrm{~h}$, respectively. As shown in Fig. 2c, for SIM and SHPEMs, the cytotoxicity increased with the prolongation of culture time. To further investigate the influence of HA on the cytotoxicity, cells were incubated with free SIM, SPEMs and SHPEMs at $8 \mu \mathrm{g} / \mathrm{mL}$ for $4 \mathrm{~h}$. After another 20 h's incubation with normal medium, the cell viability was tested and the results were shown in Fig. 2d. Specifically, the cell viability of free SIM was $64.1 \%$, slightly higher than that of SHPEMs (57.8\%) and significantly lower than that of SPEMs (86.7\%). These results suggested that HA coating on micelles facilitated cellular uptake and subsequent cytotoxicity.

To investigate the antioxidant effect, cells were treated with different formulations and intracellular ROS was detected using DCFH-DA as the probe. RAW264.7 and 


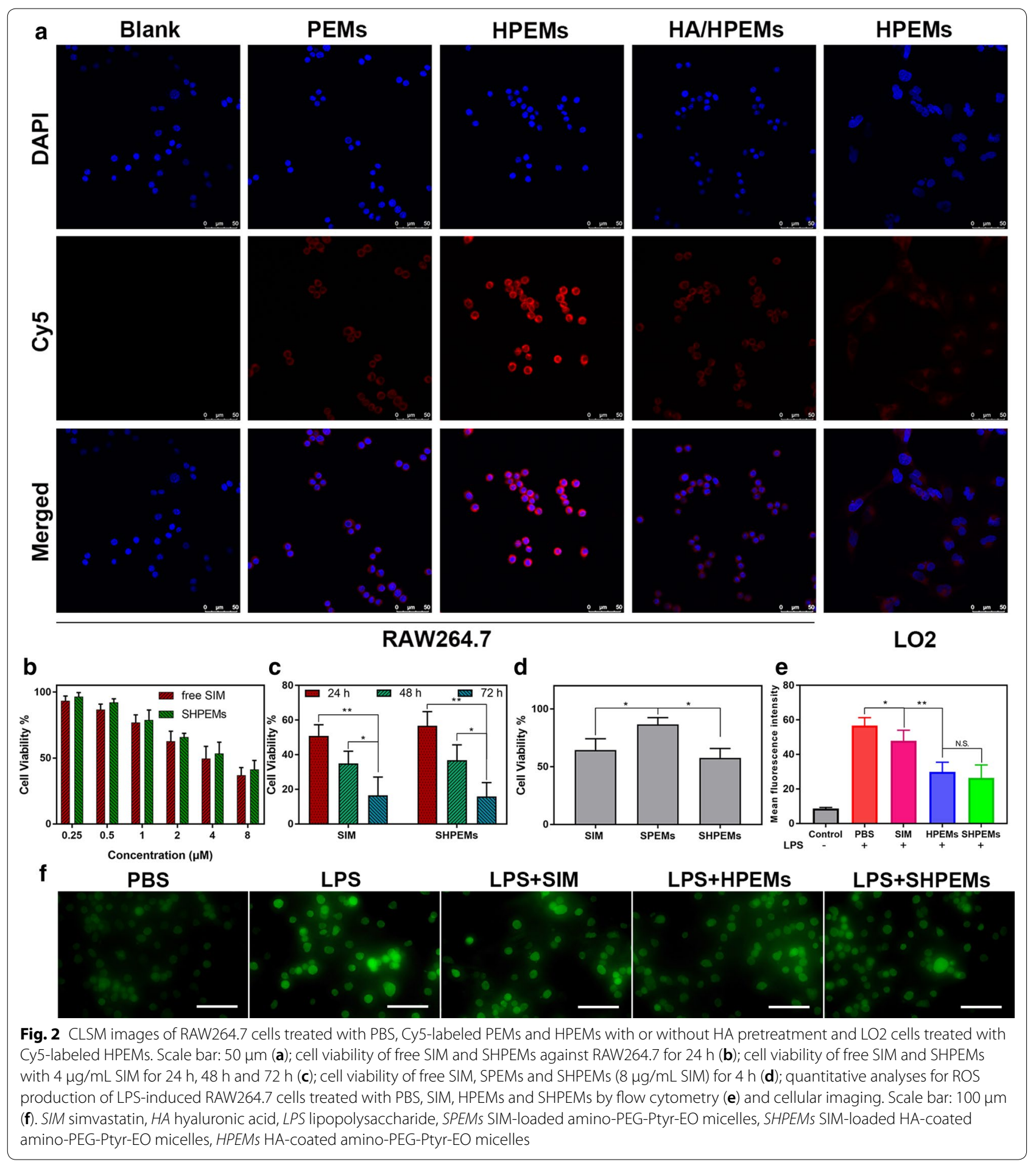

LPS-induced RAW264.7 treated with PBS were used as negative and positive control, respectively. As shown in Fig. 2e and Additional file 1: Figure S10, ROS increased significantly after cells were induced by LPS. After LPSinduced RAW264.7 cells were treated with free SIM,
ROS decreased slightly. Notably, ROS decreased dramatically after cells were treated with HPEMs and SHPEMs. Similar results can be observed from DCF fluorescence images in Fig. 2f. These results implied that HPEMs can effectively consume intracellular ROS. 


\section{Mice treatment}

Inspired by excellent cellular uptake profiles and the ideal cytotoxicity against macrophages, in vivo research was carried out to evaluate the therapeutic efficacy of SHPEMs. AopE ${ }^{-1-}$ mice were fed with a high-fat diet for 8 weeks and then randomly divided into 4 groups $(\mathrm{n}=4)$. Free SIM, SPEMs and SHPEMs were administrated through tail veil for another 4 weeks. Saline was administrated as the control group. After the experiment, the mice were sacrificed and the aortas and major organs were collected. The isolated aorta was stained with ORO shown in Fig. $3 \mathrm{a}$ and the plaque area ratio was measured as shown in Fig. 3b. The lesion area ratio of the control group with saline treatment intervention was significantly larger than that of other treatment groups. The lesion area ratio of free SIM and SPEMs was obviously smaller than the control group, indicating some therapeutic effects. Interestingly, the lesion area ratio of the SHPEMs group was significantly smaller than that of other groups, indicating enhanced anti-atherosclerotic effect. The enhanced therapeutic efficacy of SHPEMs was mainly resulted from the targeting ability derived from HA coating. Besides, the histological tissues slides of main organs in different groups were subjected to $H \& E$ staining and
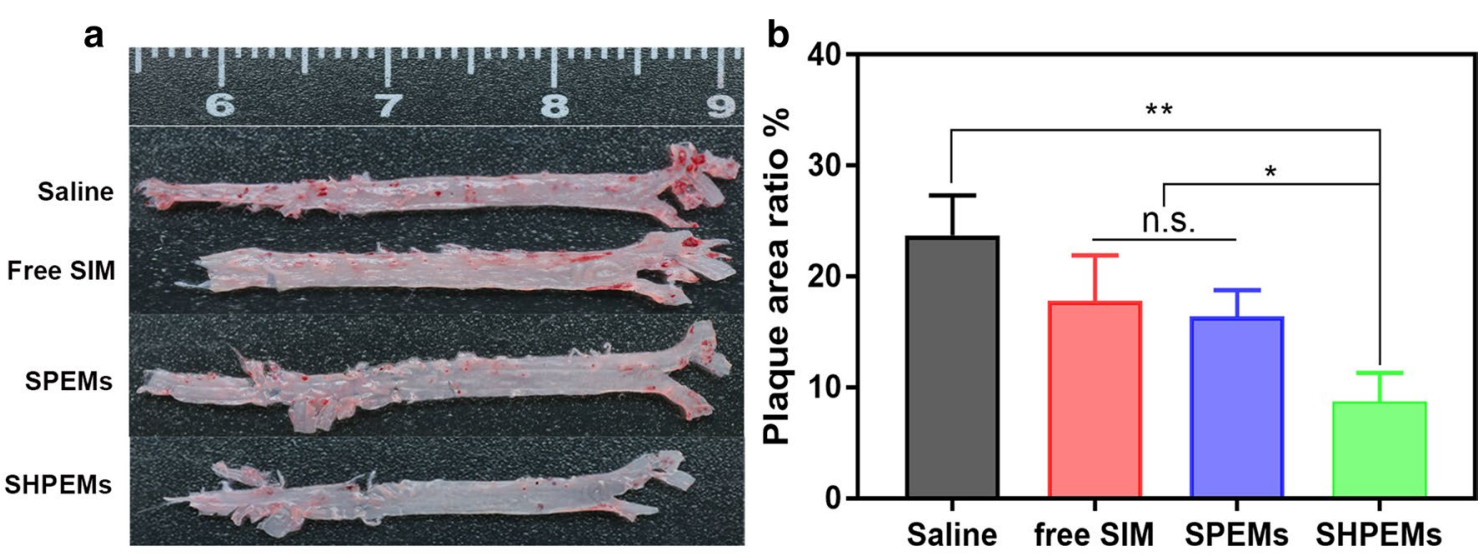

C

heart

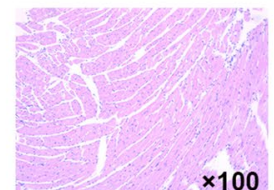

$\times 100$

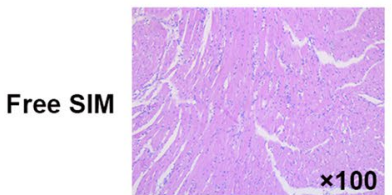

SPEMS

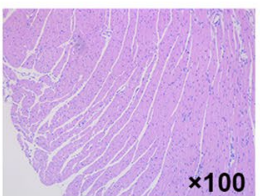

$\times 100$

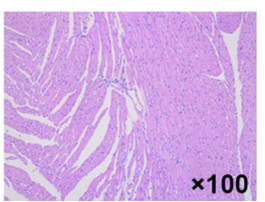

liver
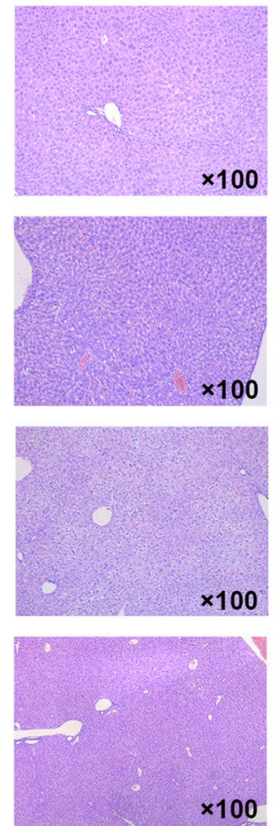

spleen
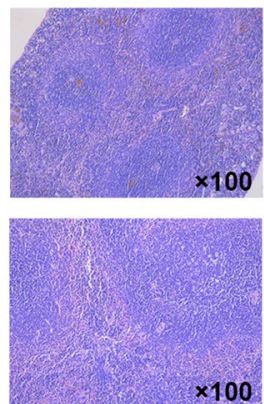

$\times 100$
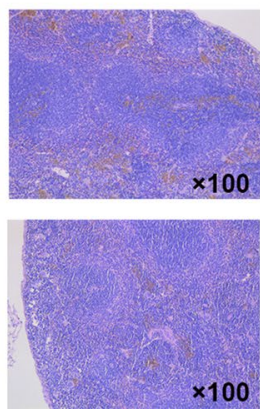

lung

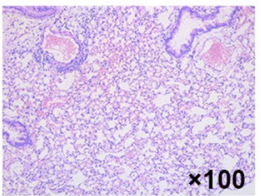

$\times 100$

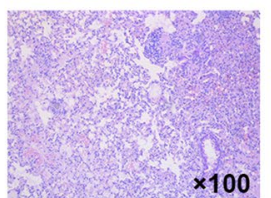

$\times 100$

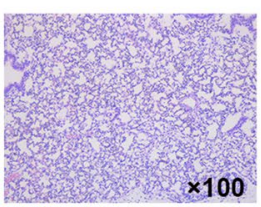

100

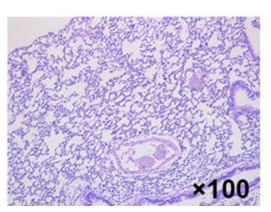

kidney
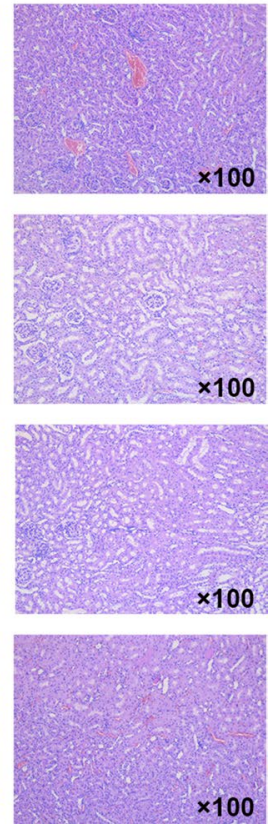

Fig. 3 Representative photographs of ORO stained aortas from each group (a); the ratio of plaque area to total area (b); H\&E staining for heart, liver, spleen, lung as well as kidney collected from mice of each group (c). SIM simvastatin, SPEMs SIM-loaded amino-PEG-Ptyr-EO micelles, SHPEMs SIM-loaded HA-coated amino-PEG-Ptyr-EO micelles 
displayed in Fig. 3c. No obviously pathological changes can be observed in heart, liver, spleen, lung and kidney, suggesting great biosafety and biocompatibility.

\section{Conclusion}

In conclusion, oxidative stress and recruitment of proinflammatory monocytes are both critical for the development of atherosclerotic plaques in the aorta. SIM possesses excellent anti-inflammatory activity, whereas the poor water solubility and low bioavailability limit its application in vivo. Therefore, an amphipathic block copolymer PEG-Ptyr-EO with oxidation sensitivity was synthesized, assembled into SIM-loaded micelles and coated with HA for targeted atherosclerosis treatment. The SIM-loaded HA-coated micelles showed excellent atherosclerotic plaque-targeted property. The in vitro tests proved that SHPEMs effectively suppressed the growth of RAW264.7 cells and significantly reduced the oxidative stress in macrophages. On the other hand, by reacting with $\mathrm{H}_{2} \mathrm{O}_{2}$, the micellar carrier itself further alleviated oxidative stress in LPS-induced RAW264.7. Consequently, the CD44-targeted and $\mathrm{H}_{2} \mathrm{O}_{2}$-responsive polymeric micelles loaded with SIM possess excellent therapeutic effects and low side effects in atherosclerosis treatment.

\section{Supplementary Information}

The online version contains supplementary material available at https://doi. org/10.1186/s12951-020-00744-w.

Additional file 1. Additional figures.

\section{Abbreviations}

SIM: Simvastatin; HA: Hyaluronic acid; PEG-Ptyr-EO: Poly(ethylene glycol)poly(tyrosine-ethyl oxalyl); ROS: Reactive oxygen species; LDL: Lowdensity lipoprotein; CVD: Cardiovascular disease; Mal-PEG-NH $\mathrm{N}_{2}$ : Maleimide poly(ethylene glycol) amine; Tyr-NCA: L-Tyrosine N-carboxyanhydride; LPS: Lipopolysaccharide; DCFH-DA: 2', 7'-Dichlorofluorescin diacetate; DMF: N, N-Dimethylformamide; DCM: Dichloromethane; DMSO: Dimethylsulfoxide; THF: Tetrahydrofuran; TEA: Triethylamine; GPC: Gel permeation chromatography; CMC: Critical micelle concentrations; $\mathrm{PBS}$ : Phosphate buffer solution PEMs: Amino-PEG-Ptyr-EO micelles; HPEMs: HA-coated PEMs; TEM: Transmission electron microscopy; SHPEMs: SIM-loaded HPEMs; DLE: Drug loading efficiency; DLC: Drug loading content; SPEMs: SIM-loaded PEMs; SPMs: SIMloaded Mal-PEG-Ptyr micelles; ORO: Oil red O.

\section{Acknowledgements}

This work was supported by the National Natural Science Foundation of China (82070366; 81720108022, B.Z., 81601539, D.M.); Supported by the Fundamental Research Funds for the Central Universities, Nanjing University (2020021414380462); The key project of Jiangsu Commission of Health (K2019025); The social development project of science and technology project in Jiangsu Province (BE2017707); Key medical talents of the Jiangsu province, the "13th Five-Year" health promotion project of the Jiangsu province (ZDRCA2016064); Jiangsu Provincial Key Medical Discipline (Laboratory) (ZDXKA2016020); the project of the sixth peak of talented people (WSN-138); the Nanjing Medical Science and technique Development Foundation (ZKX19018; QRX17057); China Postdoctoral Science Foundation (2019M661804) and Jiangsu Province postdoctoral Science Foundation (2019k060).

\section{Authors' contributions}

DM performed materials preparation and wrote the manuscript. DM, BX and BZ performed the conception and design of the study. DM and JL performed in vivo experiments. YQ and XS performed in vitro experiments. YL, Song Shen and $Y L$ performed image analyses. All authors read and approved the final manuscript.

\section{Availability of data and materials}

The datasets used and/or analyzed during the current study are available from the corresponding author on reasonable request.

\section{Ethics approval and consent to participate}

Not applicable.

\section{Competing interests}

The authors declare no competing financial interest.

\section{Author details}

${ }^{1}$ Department of Radiology, Affiliated Nanjing Drum Tower Hospital of Nanjing University Medical School, 321 Zhongshan road, Nanjing 210008, Jiangsu, China. ${ }^{2}$ Department of Cardiology, Nanjing Drum Tower Hospital, Clinical College of Nanjing Medical University, Nanjing 210008, China. ${ }^{3}$ Department of Cardiology, Affiliated Nanjing Drum Tower Hospital of Nanjing University Medical School, Nanjing 210008, China. ${ }^{4}$ Department of Cardiology, Nanjing Drum Tower Hospital, Medical School of Nanjing University, Nanjing 210008, China. ${ }^{5}$ State Key Laboratory of Pharmaceutical Biotechnology, Nanjing University, 321 Zhongshan road, Nanjing Jiangsu 210023, China. ${ }^{6}$ Institute of Brain Science, Nanjing University, 321 Zhongshan road, Nanjing 210008, Jiangsu, China.

Received: 1 October 2020 Accepted: 1 December 2020

Published online: 07 December 2020

References

1. Schiener M, Hossann M, Viola JR, Ortega-Gomez A, Weber C, Lauber K, et al. Nanomedicine-based strategies for treatment of atherosclerosis. Trends Mol Med. 2014;20:271-81.

2. Chan CKW, Zhang L, Cheng CK, Yang H, Huang Y, Tian XY, et al. Recent advances in managing atherosclerosis via nanomedicine. Small. 2018;14:1-16.

3. Alaarg A, Senders ML, Varela-Moreira A, Pérez-Medina C, Zhao Y, Tang $J$, et al. A systematic comparison of clinically viable nanomedicines targeting HMG-CoA reductase in inflammatory atherosclerosis. J Control Release. 2017;262:47-57.

4. Gao C, Huang Q, Liu C, Lee SMY, Wang R. Treatment of atherosclerosis by macrophage-biomimetic nanoparticles via targeted pharmacotherapy and sequestration of proinflammatory cytokines. Nat Commun. 2020;11:2622

5. Krolikoski M, Monslow J, Puré E. The CD44-HA axis and inflammation in atherosclerosis: a temporal perspective. Matrix Biol. 2019;78-79:201-18.

6. Duivenvoorden R, Tang J, Cormode DP, Mieszawska AJ, Izquierdo-Garcia D, Ozcan C, et al. A statin-loaded reconstituted high-density lipoprotein nanoparticle inhibits atherosclerotic plaque inflammation. Nat Commun. 2014;5:1-12.

7. Kim M, Sahu A, Hwang Y, Kim GB, Nam GH, Kim IS, et al. Targeted delivery of anti-inflammatory cytokine by nanocarrier reduces atherosclerosis in Apo E-/- mice. Biomaterials. 2020;226:119550.

8. Katsuki S, Matoba T, Nakashiro S, Sato K, Koga Jl, Nakano K, et al. Nanoparticle-mediated delivery of pitavastatin inhibits atherosclerotic plaque destabilization/rupture in mice by regulating the recruitment of inflammatory monocytes. Circulation. 2014;129:896-906.

9. Tang J, Lobatto ME, Hassing L, Van Der Staay S, Van Rijs SM, Calcagno C, et al. Inhibiting macrophage proliferation suppresses atherosclerotic plaque inflammation. Sci Adv. 2015;1:e1400223.

10. Leung PO, Wang SH, Lu SH, Chou WH, Shiau CY, Chou TC. Simvastatin inhibits pro-inflammatory mediators through induction of heme oxygenase-1 expression in lipopolysaccharide-stimulated RAW264.7 macrophages. Toxicol Lett. 2011;207:159-66. 
11. Xie Y, Liu C, Huang H, Huang J, Deng A, Zou P, et al. Bone-targeted delivery of simvastatin-loaded PEG-PLGA micelles conjugated with tetracycline for osteoporosis treatment. Drug Deliv Transl Res. 2018;8:1090-102.

12. Bejarano J, Navarro-Marquez M, Morales-Zavala F, Morales JO, GarciaCarvajal I, Araya-Fuentes E, et al. Nanoparticles for diagnosis and therapy of atherosclerosis and myocardial infarction: Evolution toward prospective theranostic approaches. Theranostics. 2018;8:4710-32.

13. Maruf A, Wang Y, Yin T, Huang J, Wang N, Durkan C, et al. Atherosclerosis treatment with stimuli-responsive nanoagents: recent advances and future perspectives. Adv Healthc Mater. 2019;8:1-28.

14. Zhang R, Liu R, Liu C, Pan L, Qi Y, Cheng J, et al. A pH/ROS dual-responsive and targeting nanotherapy for vascular inflammatory diseases. Biomaterials. 2020;230:119605.

15. Kheirolomoom A, Kim CW, Seo JW, Kumar S, Son DJ, Gagnon MKJ, et al. Multifunctional nanoparticles facilitate molecular targeting and miRNA delivery to inhibit atherosclerosis in ApoE-/- Mice. ACS Nano. 2015;9:8885-97.

16. Wei $X$, Ying M, Dehaini D, Su Y, Kroll AV, Zhou J, et al. Nanoparticle functionalization with platelet membrane enables multifactored biological targeting and detection of atherosclerosis. ACS Nano. 2018;12:109-16.

17. Wang Y, Li L, Zhao W, Dou Y, An H, Tao H, et al. Targeted therapy of atherosclerosis by a broad-spectrum reactive oxygen species scavenging nanoparticle with intrinsic anti-inflammatory activity. ACS Nano. 2018;12:8943-60.

18. Kamaly N, Fredman G, Fojas JJR, Subramanian M, Choi WI, Zepeda K, et al. Targeted interleukin-10 nanotherapeutics developed with a microfluidic chip enhance resolution of inflammation in advanced atherosclerosis. ACS Nano. 2016;10:5280-92.

19. Wu T, Chen X, Wang Y, Xiao H, Peng Y, Lin L, et al. Aortic plaque-targeted andrographolide delivery with oxidation-sensitive micelle effectively treats atherosclerosis via simultaneous ROS capture and anti-inflammation. Nanomed Nanotechnol Biol Med. 2018;14:2215-26.

20. Hou X, Lin H, Zhou X, Cheng Z, Li Y, Liu X, et al. Novel dual ROS-sensitive and $C D 44$ receptor targeting nanomicelles based on oligomeric hyaluronic acid for the efficient therapy of atherosclerosis. Carbohydr Polym. 2020;232:1-8.

21. Tapeinos C, Pandit A. Physical, chemical, and biological structures based on ROS-sensitive moieties that are able to respond to oxidative microenvironments. Adv Mater. 2016;28:5553-85.

22. Liu C, Zhu X, Wang X, Miao D, Liang X, Wang C, et al. Hydrogen peroxideresponsive micelles self-assembled from a peroxalate ester-containing triblock copolymer. Biomater Sci. 2016;4:255-7.

23. Tao W, He Z. ROS-responsive drug delivery systems for biomedical applications. Asian J Pharm Sci. 2018;13:101-12.

24. Gu X, Qiu M, Sun H, Zhang J, Cheng L, Deng C, et al. Polytyrosine nanoparticles enable ultra-high loading of doxorubicin and rapid enzymeresponsive drug release. Biomater Sci Royal Soc Chem. 2018;6:1526-34.

25. Huang J, Hastings CL, Duffy GP, Kelly HM, Raeburn J, Adams DJ, et al. Supramolecular hydrogels with reverse thermal gelation properties from (Oligo)tyrosine containing block copolymers. Biomacromology. 2013;14:200-6

26. Sill KN, Sullivan B, Carie A, Semple JE. Synthesis and Characterization of Micelle-Forming PEG-Poly(Amino Acid) copolymers with iron-hydroxamate cross-linkable blocks for encapsulation and release of hydrophobic drugs. Biomacromol. 2017;18:1874-84.

27. Liu X, Li X, Zhou L, Li S, Sun J, Wang Z, et al. Effects of simvastatin-loaded polymeric micelles on human osteoblast-like MG-63 cells. Colloids Surfaces B Biointerfaces. 2013;102:420-7.

28. Li W, Zhou C, Fu Y, Chen T, Liu X, Zhang Z, et al. Targeted delivery of hyaluronic acid nanomicelles to hepatic stellate cells in hepatic fibrosis rats. Acta Pharm Sin B. 2020;10:693-710.

\section{Publisher's Note}

Springer Nature remains neutral with regard to jurisdictional claims in published maps and institutional affiliations.
Ready to submit your research? Choose BMC and benefit from:

- fast, convenient online submission

- thorough peer review by experienced researchers in your field

- rapid publication on acceptance

- support for research data, including large and complex data types

- gold Open Access which fosters wider collaboration and increased citations

- maximum visibility for your research: over $100 \mathrm{M}$ website views per year

At BMC, research is always in progress.

Learn more biomedcentral.com/submissions 